\title{
SuperAGILE: the hard X-ray Imager for the AGILE space mission
}

\author{
M. Feroci*, E. Costa, P. Soffitta, E. Del Monte, G. Di Persio, \\ I. Donnarumma, Y. Evangelista, M. Frutti, I. Lapshov' ${ }^{1}$ \\ F. Lazzarotto, M. Mastropietro², E. Morelli³ ${ }^{3}$ L. Pacciani, \\ G. Porrovecchio, M. Rapisarda ${ }^{4}$, A. Rubini, M. Tavani, and A. Argan \\ INAF - Istituto di Astrofisica Spaziale e Fisica Cosmica - Roma \\ ${ }^{1}$ also, IKI, Academy of Sciences, Moscow, Russia \\ ${ }^{2}$ also, CNR - Istituto Metodologie Inorganiche e dei Plasmi - Area Ricerca \\ Montelibretti \\ ${ }^{3}$ INAF - Istituto di Astrofisica Spaziale e Fisica Cosmica - Bologna \\ ${ }^{4}$ also, ENEA - Centro Ricerche - Frascati \\ * Corresponding Author: INAF/IASF - Via Fosso del Cavaliere 10000133 Rome, \\ Italy - e-mail: marco.feroci@iasf-roma.inaf.it - ph. +39-06-4993-4099, fax \\ +39-06-2066-0188
}

\begin{abstract}
SuperAGILE is a coded mask experiment based on silicon microstrip detectors. It operates in the $15-45 \mathrm{keV}$ nominal energy range, providing crossed one-dimensional images of the X-ray sky with an on-axis angular resolution of 6 arcmin, over a field of view in excess of 1 steradian. It was designed as the hard X-ray monitor of the AGILE space mission, a small satellite of the Italian Space Agency devoted to image
\end{abstract}


the gamma-ray sky in the $30 \mathrm{MeV}-50 \mathrm{GeV}$ energy band. The AGILE mission was launched in a low-earth orbit on $23^{\text {rd }}$ April 2007. In this paper we describe the SuperAGILE experiment, its construction and test processes, and its performance before flight, based on the on-ground test and calibrations.

Key words: High Energy Astrophysics, X-ray Detectors, Microstrip Detectors, Coded Mask Imaging

PACS: 7.85.-m, 29.40.Wk, 95.55.Ka

\section{Introduction}

Gamma-ray astronomy is one of the least explored windows on our Universe. The intrinsic instrumental difficulty to detect and image photons with energy between few tens of $\mathrm{MeV}$ and tens of $\mathrm{GeV}$ has led to only two narrow observational periods so far, with SAS-2 (1973, Fichtel et al. 1975) and CosB (1975-1982, Bennet 1990), and with EGRET (1991-1999, Hartman et al. 1999). Originally proposed and designed to fill the gap between EGRET and

the larger, GLAST mission 1 , expected to become operational in 2008, due to significant delays the Italian AGILE gamma-ray mission is expected to observe the gamma-ray sky over the same period of time, but with a different observing strategy (Tavani et al. 2006). In addition to the observation of the "known" gamma-ray sources, the AGILE main scientific goal is the discovery of new gamma ray transients (e.g., gamma ray bursts, outbursts of AGNs, microquasars, ...) and of the counterparts of the unidentified EGRET sources (e.g., Grenier 2003). To accomplish this, the gamma-ray imager on board AGILE has a very large field of view (approximately 2 steradians) and a very

$\overline{1 \text { e.g., }}$ http://www-glast.stanford.edu/ 
good effective area and angular resolution at large off axis angles.

AGILE (Tavani et al. 2006), the first small mission of the Italian Space Agency, was launched from the Shriharikota base in India with a PSLV launcher 2 on $23^{r d}$ April 2007 in an equatorial orbit $\left(\sim 2^{\circ} .5\right.$ inclination $)$ at $\sim 545 \mathrm{~km}$ altitude. The $\sim 350 \mathrm{~kg}$ AGILE satellite carries a $\sim 120 \mathrm{~kg}$ scientific payload composed of a Gamma Ray Imaging Detector (GRID) - a silicon microstrip tracker and a CsI "mini" calorimeter, surrounded by a plastic anticoincidence - and a hard X-ray monitor, SuperAGILE. In fact, despite the improved efficiency of the gamma-ray imager with respect to its predecessors, the observation of celestial gamma-ray sources suffers severe photon starvation and angular confusion. The simultaneous observation of the gamma-ray sources with an instrument operating in hard X-rays, an energy range where higher sensitivity and better angular resolution can be achieved, helps with the identification of the source, with the very important benefit of allowing to correlate and study the simultaneous emission in two distant energy bands. This is indeed the basic scientific justification for having an experiment like SuperAGILE onboard the gamma-ray mission AGILE.

SuperAGILE is a coded mask experiment, operating in the nominal energy range 15-45 keV, imaging a field of view in excess of 1 steradian with a 6 arcminutes one-dimensional angular resolution (on axis), in two orthogonal directions in the sky. The main scientific goal of SuperAGILE is to provide a prompt, unambiguous and accurate identification of the gamma-ray sources observed with the GRID. Based on what we know today, this is a task that can be accomplished on a relatively small sample of sources (e.g., gamma-ray

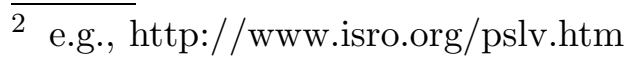


pulsars, blazars, gamma-ray bursts). However, with a sensitivity of $\sim 15 \mathrm{mCrab}$ (in $50 \mathrm{ks}$ of observation) and field of view of $107^{\circ} \times 68^{\circ}$ (at zero response), SuperAGILE will also operate as a (quasi) all sky monitor of the X-ray sky, allowing the monitoring of known Galactic sources (low and high mass X-ray binaries, magnetars, ...) and possibly the discovery of new transient sources.

This paper describes the concept and details of the design and construction of the flight model of the SuperAGILE experiment, providing the reference performance and calibrations obtained before launch. A Detailed description of the ground calibration procedures and results will be given in a future publication.

\section{The Concept and the Context}

SuperAGILE (SA) was conceived as a monitoring instrument for the primary mission experiment, GRID. As such, the first requirement was to use minimal resources and do not significantly affect the scientific response of GRID. This was a major constraint to the SA design and performance. The operating principle of GRID is based on the conversion of gamma-ray photons in electron-positron pairs by thin tungsten sheets, and tracking the pairs, in energy and direction, by microstrip silicon detectors, thus reconstructing the kinematics of the impinging photon.

Since to absorb X-rays a thin amount of matter is sufficient, the only possible location for SA, compatible with the small dimension of the AGILE payload and with the scientific request of a co-aligned field of view (FOV) for the two instruments, is on top of GRID. Thus, any mass of the SA experiment will 
itself acts as an unwanted converter of gamma-ray photons, resulting in the decrease of the GRID efficiency and in the increase of its particle background. This brought to the use of materials with low atomic number and keep the weight at minimum, to decrease the conversion probability for photons. The residual (average) probability of SA to convert a gamma-ray photon, thus decreasing the signal to noise of GRID, was then kept at less than 10\%. For the same reasons, SA had to be located inside the anticoincidence surrounding the GRID. This implies that a $5 \mathrm{~mm}$ thick layer of plastic scintillator, with its support structure, completely obstructs the SA FOV, largely decreasing its efficiency, especially at energies lower than $20 \mathrm{keV}$ (e.g., its transparency on axis is $70 \%$ at $15 \mathrm{keV})$.

SA had then to satisfy the scientific requirements relevant to its monitoring function. First, covering the largest fraction of the GRID FOV ( $\sim 2$ steradian $)$ was obtained by co-aligned FOVs. Then, a trade-off was necessary on the size of the FOV: contrary to the gamma-ray band, at X-rays the main source of background for an instrument with a large FOV are X-ray photons of the isotropic diffuse X-ray background, undistinguishable from the good source photons, at detection level. Thus, the larger is the FOV, the lower is the experiment sensitivity and the larger is the telemetry required to transmit individual events to ground. The target sensitivity was therefore set to $\sim 10$ 15 mCrab $5 \sigma$ in one day $\left(1 \mathrm{mCrab}\right.$ corresponds to $\sim 4 \times 10^{-4}$ photons $\mathrm{cm}^{-2} \mathrm{~s}^{-1}$ or $\sim 1.5 \times 10^{-11}$ erg $\mathrm{cm}^{-2} \mathrm{~s}^{-1}$ in $15-45 \mathrm{keV}$ ), a level adequate to the arcminlevel angular resolution, with respect to the source confusion limit. Taking into account the fixed geometry of the detectors, the choice for the FOV is a rectangle, $107^{\circ} \times 68^{\circ}$ at zero response, for each pair of detectors. The two pairs of detectors are then oriented at approximately $90^{\circ}$, so that $\mathrm{SA}$ can provide 
the two coordinates of sources, when occurring within the central $68^{\circ} \times 68^{\circ}$ part of the FOV, and still provide the X-ray detection of a gamma-ray source and its single coordinate when at the edges of the FOV. The mask-detector distance was instead chosen such that the source location accuracy is in the range of few arcminutes, thus useful to follow-up the SA localizations with other observatories, at X-rays and other wavelengths. On the other hand, the systematic error in the satellite attitude reconstruction is expected in the range 1-2 arcminutes, thus we expect to reach the systematics for most sources.

Finally, to keep the development, manufacturing and qualification costs at minimum, SA employs the same constructive elements as the GRID. In particular, the silicon detectors, the ASIC model, the high voltage power supply, the carbon fiber detector tray technology are just the same as the GRID.

Figure ?? shows a sketch with an exploded view of the SA experiment, separating the detection plane, the collimator and the mask. In Table 1 the main instrument parameters are summarized. The development of the SA flight model was preceded by a series of tests on prototypes and by the construction and test of an engineering model. Details on them may be found in Soffitta et al. (2002) and Feroci et al. (2004) and references therein.

\section{The Detection Plane}

The Detection Plane (DP) is the sensitive unit of SA, composed of the detector tray on which the SA Detection Units (DUs, silicon detectors plus front-end electronics) are fixed.

The detector tray is a $44 \mathrm{~cm} \mathrm{x} 44 \mathrm{~cm} \mathrm{x} 0.5 \mathrm{~cm}$ support plane made of aluminum 
honeycomb, covered with two outer layers of carbon fiber tissue. To prevent X-ray background photons to reach the detectors from below, in the area where the GRID material does not ensure enough shielding, a $120 \mu \mathrm{m}$ thick tungsten frame was glued to the back side of the tray. The total weight of the tray is $\sim 850$ g. A special care was devoted to its planarity, required by the gluing process of the DUs, that was requested and obtained within $0.15 \mathrm{~mm}$ overall. The detector tray was manufactured by Plyform (Italy) and Oerlikon Contraves Italia.

\subsection{The Silicon Microstrip Detectors}

The sensitive elements of the SA experiment are single-sided, AC-coupled, ntype, p-strip silicon microstrip detectors manufactured by Hamamatsu (Japan). Each of the $4 \mathrm{SA}$ detectors is composed of 4 Silicon tiles, $95 \mathrm{~mm}$ x $95 \mathrm{~mm}$ x $0.41 \mathrm{~mm}$ in size, implanted with a pattern of $76860 \mu \mathrm{m}$ wide metal strips at $121 \mu \mathrm{m}$ pitch. The strip pattern is surrounded by a guard ring and a bias ring. The active area of each tile is thus approximately $84 \mathrm{~cm}^{2}$, providing an active area of about $336 \mathrm{~cm}^{2}$ per detector. Each detector was assembled first by gluing two tiles head-to-head to form one ladder. Then, two ladders were independently glued side by side, with a controlled separation, onto a $\mathrm{Cu} / \mathrm{Au}$ grid-coated kapton foil (hereafter "flexbias"), also used to bring the electrical positive bias ( $\sim 90 \mathrm{~V})$ from the SAFEE (SuperAGILE Front End Electronics) boards to the Si detectors. The electrical contact between the back of the Si tiles and the flexbias is guaranteed by 5 spots of a conductive epoxy glue per tile, while the mechanical stiffness of the system is provided by a larger number of spots of silicon glue. 
The mechanical assembly was performed giving special care to the alignment issues. The head-to-head assembly was such that the individual strips are phased within $5 \mu \mathrm{m}$ and parallel to better than 10 arcsec. The mechanical distance between the two ladders was then fixed such that the separation between the innermost active strips of the two ladders is equivalent to the smallest integer number (18) of strip pitches, in order to keep the impact of the mechanical separation on the coded imaging capability at minimum. This goal was achieved to within $\sim 10 \mu \mathrm{m}$, also thanks to the excellent mechanical quality of the mask and cuts of the Hamamatsu silicon tiles.

The strips of the two tiles composing one ladder are bonded one-to-one by 25 $\mu \mathrm{m}$ Aluminum wires, so that effective 19-cm long strips are obtained, whose capacitive load is $30 \mathrm{pF}$. Each of these strips is connected to the bias ring through a parallel of two $40 \mathrm{M} \Omega$ on-chip poly-resistors. Bias and guard rings are grounded.

Further details about the detector assembly procedure, carried out by the MIPOT company (Italy), may be found in Rapisarda et al. (2004).

\subsection{The SuperAGILE Front End Electronics (SAFEE)}

Each of the 4 SA detectors is read-out through an independent SuperAGILE Front-End Electronics (SAFEE) unit. The "core" of the SAFEE are its 12 XAA1.2 ASIC chips, each of them including 128 independent spectroscopic chains, composed of preamplifier, discriminator, amplifier and peak stretcher. Each detector strip is connected to one of the 128 channels of one XAA1.2. The pitch of the detector $(121 \mu \mathrm{m})$ was adapted to the $96 \mu \mathrm{m}$ pitch of the 
XAA1.2 by means of a pitch adapter (gold tracks deposited on a two layer alumina board). Connections between detector strips and pitch adapter and between the control pads of the ASIC and the SAFEE PCB are made by 25 $\mu \mathrm{m} \mathrm{Al}$ wire bondings. Connections between the pitch adapter and the ASIC input channels are made by $17 \mu \mathrm{m} \mathrm{Al}$ wires.

A detailed description of the SAFEE is given in Pacciani et al. (2007), whereas the XAA1.2 characteristics and performances may be found in Del Monte et al. (2007). Here we just summarize the major features of these systems.

Each SAFEE is both conceptually and electrically organized in 4 almost independent Daisy Chains (DCs), including 3 ASIC dies each. When in DC, the chain of three XAA1.2 chips acts as it was a single chip. In fact the 12 biases requested to configure each XAA1.2 chip are in common for the 3 elements in one DC, with a "winner takes it all" logic (meaning that a triggered channel disables all the others until after complete processing) applies to the 384 channels of a DC. Upon trigger, the relevant DC places on its output bus the following analog information, in differential current signals: multiplicity signal (allows to select triggers from single and multiple strips), amplitude, group address (most significant bits of the detector strip address, between 1 and 12) and strip address (least significant bits, between 1 and 32). These signals are directly passed to the 144-pin connector interfacing the SAFEE to its SAIE (SuperAGILE Interface Electronics, see \$6). The dead time of each DC depends on the setting of three parameters of the XAA1.2, determining the delay from the discrimination to the start of the "output-ready" gate (TrigDelBias), the duration of the gate (TrigWidthBias) and the time to the chip reset (ResWidthBias). At the AGILE launch the total deadtime per DC was set as about $80 \mu \mathrm{s}$, with few $(\sim 5-10)$ microseconds variations from channel to chan- 
nel and $\sim 10 \mu$ s from ASIC to ASIC. This long "analogue" deadtime makes it negligible the processing time of the A/D converter in the SAIE, that runs in parallel to the chip reset time. It is worth noticing that the event processing from the 16 SA DCs is independent and multiplexed in the SAIEs, thus the overall instrument operational deadtime can be conventionally defined as 1/16 of that of the individual DC, that is $\sim 5 \mu \mathrm{s}$.

Only 8 of the 12 configuration biases of the XAA1.2 were made programmable by telecommand. To this purpose, 4 DAC octal chips were used, one per DC, whose reference voltage is provided by the regulated $\pm 2 \mathrm{~V}$ lines powering the ASICs themselves. In this set-up, the biases are automatically dumped off at the power supply switch off.

The dimension of the SA tray were constrained by external parameters (e.g, GRID design and internal dimensions of the anticoincidence subsystem). Therefore the electronic components that were required for a SAFEE could not all fit on a single printed circuit board (PCB) lying on the same plane as the detector. Still, the ASICs had to be wire-bonded to the detector strips and lay close to them. Therefore, the SAFEE was designed as a 6-layer rigid-flex PCB, with two rigid parts connected by a flexible part. The part hosting the ASICs and the bias resistors was placed horizontally near to the detector, while the DACs and their circuits are hosted on a larger rigid part, screwed to the external wall of the collimator (see photo in Figure ??). The vertical SAFEE is protected by a custom aluminum box.

Due to the required density of the SAFEE circuit and to the low-cost approach of the AGILE mission, most of the electronic components - including the XAA1.2 - are commercial grade, except for resistors that are MIL-standard. 
In order to ensure a minimum level of quality assurance, a few environmental tests were carried out. In particular, the XAA1.2 were radiation tested for Latch-up, Single Event Upset (SEU) and Dose effects at the ion accelerator facility of INFN in Legnaro (Del Monte et al., 2005). The DACs (Analog Device's AD8842AR) were tested for latch-up and SEU by the Hirex Engineering company (France). Finally, each complete SAFEE underwent a 10-day burn-in test at $75^{\circ} \mathrm{C}$. Further environmental tests (thermal-vacuum, acoustic, EMC, vibrations) were carried out at higher levels of system integration (detection plane, experiment, payload and satellite). All of the above tests were successful, and from them no warning was derived for the operations in orbit. However, for further safety, the power supplies lines of both the XAA1.2 $( \pm 2 \mathrm{~V})$ and the AD8842AR $( \pm 5 \mathrm{~V})$ were equipped with autonomous latch-up protection circuits onboard.

The power consumption of the XAA1.2 was one of the issues of the ASIC optimization (the XA1.3 version used $\sim 3 \mathrm{~mW} /$ channel). In our baseline configuration, the XAA1.2 requires $\sim 1 \mathrm{~mW} /$ channel. Changes to the setting of the biases controlling the analog signal shape can bring to changes to this value. A back-up solution is always represented by the exclusion of the stretcher circuit, allowing a decrease in power consumption by a factor more than 2. Considering the consumption on the $5 \mathrm{~V}$ and $130 \mathrm{~V}$ lines, the total power requested by the entire SA DP (excluding only the SAIE boards described below) is less than $7 \mathrm{~W}$ overall. Further details may be found in Pacciani et al. (2007) and Del Monte et al. (2007). 


\subsection{Integration and Alignment of Detection Units}

One SA Detection Unit (DU) is composed of one detector (that is, 4 Silicon tiles), one SAFEE and their connecting $\mathrm{Cu} / \mathrm{Au}$ coated kapton board, the socalled "flexbias". The horizontal section of the SAFEE board is glued on the

flexbias already connected to the detector by conductive glue. The shape of the two is such that the high voltage contact on the SAFEE board can be brought, by soldering, to the flexbias and to the two individual ladders of the detector thereafter. The mechanical connection between the detector and the SAFEE PCB is then reinforced with head-to-head spots epoxy gluing. Finally, the detector strips and SAFEE input channels are interconnected through $25 \mu \mathrm{m} / 17 \mu \mathrm{m}$ aluminum wire bonding, thus completing a SA Detection Unit.

\subsection{Integration and Alignment of the Detection Plane}

After proper testings (see \$9), the 4 SA DUs were aligned and integrated on the detector tray. For the alignment of the experiment the following approach was used: have the internal alignment of the masks and DUs systems to better than half of the overall alignment requirement and leave only the degree of freedom of aligning the two complete systems one to the other. The scientific requirement on the overall detector-mask alignment was set as half of detector strip $(60 \mu \mathrm{m})$ over its total length $(190 \mathrm{~mm})$ (corresponding to an angle of $\sim 60$ arcsec). In fact, the main effect of a mask-detector misalignment is the loss in the signal-to-noise ratio of the reconstructed source (e.g., Charalambous et al. 1984), due to the fraction of the encoded photons detected by the "wrong" detector strip. In practice, this effect becomes important (that is, of the order 
of few percent) when the misalignment is larger than half of the mask element width. For SA, this condition implies a linear displacement of $121 \mu \mathrm{m}$, thus our requirement of $60 \mu \mathrm{m}$ corresponds to half of the error budget on the alignment.

The DUs were spot-glued directly onto the carbon fiber detector tray. As a consequence, their alignment had to be obtained simultaneously to their integration. An accurate mechanical reference system was obtained by tightly inserting 3 steel pins with very precise cross-shaped engravings (allowing a resolution of $\sim 5 \mu \mathrm{m}$ ) in the outer frame of the tray. From this point onward, these 3 points represented the "absolute" reference frame of the SA DP, in which the ideal geometrical positions of each DU were predetermined. Each DU, supported by a vacuum plier, was then slowly vertically dropped to its position onto the pre-glued detector tray underneath, whose position could be adjusted with micrometric resolution at the focus of the camera of a metrology machine. The DU was kept in its position for more than 24 hours (the silicon glue polymerization time) by mechanical constraints and its position measured again afterwards. This procedure was subsequently repeated for the 4 DUs. The experimental results obtained with this procedure can be expressed in terms of parallelism and orthogonality between the innermost edges of the different DUs, and their absolute distance. The average parallelism between the 4 units is 15.7 arcsec, with a dispersion of 10.4 arcsec. The average orthogonality is 9.5 arcsec, with a dispersion of 9.4 arcsec. The average distance (nominal value $4.000 \mathrm{~mm}$ ) is $4.014 \mathrm{~mm}$, with a dispersion of $7 \mu \mathrm{m}$. The final real DP alignment is thus well within the requirement of 60 arcsec.

Further details about the DP integration and alignment procedure, carried out in collaboration with the MIPOT company, may be found in Rapisarda et al. (2004). The stability of the alignment, within the requirements, across 
the environmental tests (thermal-vacuum and vibrations) was verified at the engineering model level (Feroci et al. 2004).

\section{The Collimator and Mask System (CMS)}

The SA collimator has the double function of limiting the field of view and be an accurate mechanical support for the coded mask system. Due to the constraints on trasparency (see \$1), the collimator structure is in $0.5 \mathrm{~mm}$ thick carbon fiber. To the purpose of shielding the hard X-rays of the diffuse X-ray background, the collimator walls are covered with tungsten sheets $\sim 120$

$\mu \mathrm{m}$ thick, that guarantee an absorption greater than $90 \%$ for photon energies lower than $40 \mathrm{keV}$, in the orthogonal direction.

The field of view (FOV) was determined as a trade-off between the main scientific requirements (coverage of the largest fraction of the GRID observing angle, peak 1-day sensitivity better than 15 mCrab, arcmin-level angular resolution) and the mechanical (especially volume and mass) and telemetry constraints $(25 \mathrm{kbit} / \mathrm{s})$. The chosen solution of a crossed rectangular field, $107^{\circ} \times 68^{\circ}(\mathrm{FWZR})$ covers approximately half of the GRID FOV with an expected sensitivity better than $50 \mathrm{mCrab}$ (one day integration) in the central $60^{\circ} \times 60^{\circ}$ (peaking at $10-15 \mathrm{mCrab}$ on axis), and with an average telemetry occupation of $\sim 20 \mathrm{kbit} / \mathrm{s}$. (See $\$ 13.1$ for a more extended discussion of the experiment sensitivity.)

The above FOV is achieved by collimator cells having the same size of the detector, that is $190 \mathrm{~mm}$ x $190 \mathrm{~mm}$, evenly divided in two by a sect placed orthogonal to the detector strip direction. In this way the coding direction 
has a FOV limited to $\pm 53.5^{\circ}$, while the non-coding direction extends to $\pm 34^{\circ}$. The collimator response is triangular in both directions. The collimator cell height is $142 \mathrm{~mm}$, and this also fixes the mask-detector distance, since the coded mask is supported by the $0.5 \mathrm{~mm}$ thick carbon fiber top coverage of the collimator itself. In Figure ?? the Flight unit of the SA collimator is shown as seen from the bottom, where the cells are open and visible.

The radiation blockage of the collimator is guaranteed by the Tungsten sheets glued to its walls. The sheets placed onto orthogonal walls could not arrive to touch. The corners between walls were then covered by a specially manufactured Tungsten-loaded glue, ensuring approximately the same shielding as the Tungsten sheets. The interface between the collimator and the DP was instead covered by a $135 \mu \mathrm{m}$ thick lead-tape. Finally, since the carbon fiber and the glue are not very good electrical conductors, thin copper plates (clearly visible in Figure ??) were glued onto the critical parts of the collimator walls, ensuring the electrical grounding of the entire structure.

\subsection{The Physical Design of the Coded Masks}

The SA experiment has, from the point of view of the coded mask systems, a simple configuration (e.g., in 't Zand 1992), that is mask and detector have approximately the same size. Different configurations were excluded by the mechanical and/or scientific constraints. The two independent units per coordinate allowed to manufacture the SA mask of the homologous detectors in a mirror configuration, that is, any part of the sky is seen through the mask and anti-mask code, one per detector. This strategy is expected to be highly rewarding when spatial inhomogeneities are present in one detector. With such 
a large FOV, the most common case is the partial occultation of the FOV by the Earth. In Figure ?? a simulated application of the mask/anti-mask technique shows the advantage of combining the images of the two homologous detectors when the background is not uniform (here we show the case of Earth blockage of a fraction of the field FOV).

The mask/anti-mask technique imposes a mask open fraction of 50\%, although in background-dominated experiments as SA smaller open fractions may result in better sensitivity (e.g., in 't Zand et al. 1994) and, consequently, in a saving in the telemetry resources. This open fraction also allows to use an Hadamard sequence for the source code, thus benefiting of an almost side-lobe free response function for on axis point sources. Our 787-element sequence was thus generated by means of the quadratic residue algorithm, then conceptually rounded to 788 elements to match the perfect $50 \%$ open fraction, with the addition of one element. The size and number of mask elements was driven by the highest angular resolution matching a minimum factor of 2 detector oversampling for each mask element (e.g., in' t Zand 1992 and references therein). Further details on the mask code selection and properties for SA, with numerical and Monte Carlo testing of this and alternative solutions, may be found in Lapshov et al. (2007).

\subsection{Manufacturing of the Coded Mask System}

At the manufacturing stage, the coded mask of SA, as any other, deviates from the ideal design under many respects. The major issue in the manufacturing of the SA coded mask system was doing $242 \mu \mathrm{m}$ narrow cuts on single a $440 \mathrm{~mm}$ $\times 440 \mathrm{~mm}$ wide, $117 \mu \mathrm{m}$ thick tungsten sheet, satisfying a $\sim 10-15 \mu \mathrm{m}$ accuracy 
on both the mask elements width, as well as their position and parallelism with respect to a reference direction, with the additional requirement of avoiding systematic displacements in the absolute position of the strips.

Several techniques were tested to comply with the above requirements. The chemical etching was proven on prototypes to satisfy the basic requirements and selected for the manufacturing of the engineering and flight units by the Oerlikon Contraves Italia, in cooperation with Vaiarelli s.r.l. (Milan). The result of fabrication is rather satisfactory. A sample of $\sim 400$ strips (intentionally manufactured on the same Tungsten sheet used for the flight unit) shows an average deviation from the nominal width of $0.2 \mu \mathrm{m}$, with a standard deviation of $8.0 \mu \mathrm{m}$. Very good results were also obtained with respect to the meniscus along the mask thickness: the same sample shows deviations within 5-10 $\mu \mathrm{m}$ from orthogonality. Despite these very good results (a definite technological challenge considering the wide dimension and small thickness of the tungsten sheet), a couple of issues were not fully satisfactory. The deviation of the strip widths was slightly systematic, resulting in an overall dimension of the mask approximately $50 \mu \mathrm{m}$ wider than the nominal. Although this implies only a contribution of less than $1 \mu \mathrm{m}$ per strip (less than $0.5 \%$ of the width), it also implies that the outermost narrow strips are displaced by $1 / 4$ of their width. Another important point is that the process of gluing the mask onto the $500 \mu \mathrm{m}$ thin carbon fiber top layer of the collimator brought to some arclike distortions in the linearity of several strips. The amount of this effect was however limited by the 3 strong-backs supporting the strips every $\sim 4.5 \mathrm{~cm}$. We developed software techniques to account for both defects when simulating the source shadowgram during the IROS procedure (see $\S 12$ and Lapshov et al. 2007 for details). The appearance of the CMS before integration with the 
DP is as shown in Figure ??.

\section{Mechanical Integration and Alignment}

After the individual "internal" integration of both DP and CMS, the maskdetector alignment for each DU was thus transposed to the alignment of the two above systems as rigid bodies. The alignment was carried out using a $0.1 \mu \mathrm{m}$-precision metrology machine equipped with an optical camera. A finestep movement system allowed to shift the CMS with respect to the DP. Before putting the CMS in place, reference points on the silicon detectors were targeted and saved into the memory of the metrology machine. The CMS was then positioned making its reference points to coincide as much as possible with the relevant points on the DP.

Since the rotation between the detector and mask strips is the most critical issue of our optical system, the quality of the alignment can be evaluated in terms of the angle between these two items, for each DU. This measurement was carried out, using the same metrology machine, taking the average strip direction with 8 points per detector strip and 9 points per mask strip. Considering that the detector is composed of two independent ladders (§??), we studied the misalignment independently for the two outermost detector strips of each detector, representative of the two different ladders (the very small misalignment between the two tiles of a ladder is already averaged by the multiple-point description of the strip). In Table 2 we show the final results for the two outermost strips of each detector, as well as their averages. It is worth noticing that these measurements include all the potential mechani-

cal errors in the internal alignment of our optical system: the tiles position 
within one detector, the misalignment from one detector to another, and the misalignment of the strips within the coded mask. In particular, taking into account that the alignment was basically done between rigid bodies, the larger misalignment in D2 is indicative of an intrinsic misalignment of that specific detector/mask system.

Due to the tight schedule of the production of the AGILE Flight Model, no metrology measurements could be carried out after the environmental tests (especially vibrations) because this would have required to disassemble the experiment and qualify it once again. We then rely on the tests carried out on the SA engineering model, completely representative of the flight unit from the mechanical point of view, for which we showed that after the vibration tests the internal alignment of SA was preserved to within $\sim 50$ arcsec (Feroci et al. 2004).

The above results concern the alignment internal to the SA experiment. To the purpose of relating the SA optical axes to the reference frame of the Star Trackers onboard the AGILE mission, we positioned a set of 3 optical cubes on the top corners of the mask. The orthogonality between the faces of the optical cubes is certified by the manufacturer to better than 3 arcsec. The faces of these cubes were then measured with the same machine as the SA optical axes thus allowing to determine the rotation matrix between the SA Detection Units and the Star Trackers (also equipped with optical cubes). The alignment between these two systems was measured after the integration of SA into the AGILE Payload by means of a system of theodolite and laser tracker instruments, with an angular uncertainty smaller than 5 arcsec and an absolute uncertainty smaller than $10 \mu \mathrm{m}$. Only a reference cube on the AGILE Bus and those on the Star Sensors remained optically accessible after 
the satellite integration. Their alignment was measured again after the satellite environmental tests (vibration, thermal-vacuum, EMC and acoustic) in order to verify its stability across odd environmental conditions. In this set-up only the laser tracker could be used as a measurement system, not optimal due to the small surfaces involved. The result of the two measurements shows that the alignment is preserved to within the uncertainty of the measurement, corresponding to less than $\sim 2$ arcmin.

After its complete integration, the weight of the complete SA experiment (DP and CMS, excluding SAIEs and harness) was measured as $5482 \mathrm{~g}$.

\section{The SuperAGILE Interface Electronics (SAIE)}

The SA Interface Electronics (SAIE) is the digital electronics in charge of interfacing the SAFEE units with the Payload Data Handling Unit. SA is equipped with two independent and identical SAIE boards, each one controlling two SAFEEs, one per coding direction, providing a full redundancy. A detailed description of the SAIE functionalities may be found in Pacciani et al. (2007). Here we recall its main functions:

- Regulate power supplies received from the AGILE Power Supply Unit for the SAFEE: $+2 \mathrm{~V}$ and $-2 \mathrm{~V}($ from $\pm 2.7 \mathrm{~V}$ ) independently on each Daisy Chain, $+5 \mathrm{~V},-5 \mathrm{~V}$ and +90V (from $130 \mathrm{~V}$ ) independently for each SAFEE.

- Latch-up control, with automatic switch-off, for the $+2 \mathrm{~V},-5 \mathrm{~V}$ and $+5 \mathrm{~V}$ lines, by current monitoring.

- Providing digital controls for the SAFEE DACs (for setting the XA biases).

- Storing and providing configuration strings for XA Reg-In registers. 
- Current-to-voltage conversion of amplitude, strip address, group address and multiplicity signal from each Daisy Chain.

- Assigning 12-bit differential time ( $2 \mu$ s resolution) to each event. If no event is received within $8.192 \mathrm{~ms}$, a Dummy Event is generated to preserve the sequence. The time assignment is based on a $1 \mathrm{MHz}$ clock and the GPS pulse per second signal received from the PDHU.

- Generating 36-bit Absolute Time Events, as reference to the event differential time.

- Handling simultaneous events on different Daisy Chain.

- Applying analog upper thresholds to amplitude signals.

- Applying analog upper and lower thresholds to multiplicity signals (MGO).

- Handling veto signals from other AGILE subsystems (Silicon Tracker, Minicalorimeter, Top and Lateral Anticoincidence).

- 12-bit A/D conversion of all signals.

- Conditioning temperature sensors (a total of 34 on SA).

- Building analog housekeepings (sampling time $16 \mathrm{~s}$ ): voltages, detector bias currents, temperatures.

- Building digital ratemeters (integration time $16 \mathrm{~s}$ ): total triggers, vetorejected counts, energy and multiplicity windows-rejected counts, good events.

- Generating the electronic calibration procedure: a sequence of up to 256 pulses for up to 4 amplitudes for every XA channel; the procedure can work automatically for all the 384 channels in a Daisy Chain, independently for each Daisy Chain.

- Generating the threshold scan procedure: a sequence of electrical calibrations interleaved with threshold setting variations; the procedure can work automatically for all the 384 channels in a Daisy Chain, independently for each Daisy Chain. 
The SAIE boards register each event (Good Event, Calibration Event, Dummy Event or Absolute Time Event) in a 60-bit word, containing its strip and group address, amplitude, and differential time. These events are stored in a FIFO (containing up to 16 events, 1 kbyte) waiting to be read-out by the PDHU.

Each SAIE board is encased in an Aluminum box, $380 \mathrm{~mm}$ x $202 \mathrm{~mm}$ x 20 $\mathrm{mm}$, for a total weight of $\sim 1.5 \mathrm{~kg}$ per SAIE. The power consumption of each SAIE is $\sim 3.8 \mathrm{~W}$. The 2 SAIE boards are vertically placed on two sides of the silicon tracker.

\section{The Payload Data Handling Unit}

In addition to the "standard" Data Handling operations, the AGILE Payload Data Handling Unit (PDHU, based on the 32-bit TEMIC TSC21020F DSP processor), performs a few but important functions specific to the SuperAGILE experiment. They can be summarized as follows:

- For Good Events type, converts the 12-bit group and 12-bit strip addresses into a single 13-bit "digital" address, through a Look-up Table (LUT). Four LUTs are stored in the PDHU memory (to account for variations due to temperature, power supplies, and other environmental effects) and their switch is selected by telecommand.

- Formats the 60-bit SA events (all types) received by the SAIEs into 32-bit telemetry events, and creates telemetry packets.

- Provides the 12 most significant bits to the event arrival time, bringing it from 36-bit to 48-bit (On Board Time, based on a reference time received every $1 \mathrm{~s}$ from the onboard GPS). 
- Performs the SA Burst Search and Imaging procedure (see $§$ 7.1).

- Builds the SA scientific ratemeters: 24 counters (counts every $0.5 \mathrm{~s}$, in each ladder, in 3 programmable energy ranges).

- Generates the automatic switch-off command for the relevant SA detector if a latch-up is detected from the SAIE.

- Manage the telemetry tables: as a baseline up to $25 \mathrm{kbit} / \mathrm{s}$ are available to SA (corresponding to roughly 800 counts/s, on average over the orbit).

The SA-SAIE-PDHU system was designed in order to be able to handle impulsive SA total rates as high as $100 \mathrm{kHz}$ (corresponding to a transient source more than 1500 times brighter than the Crab source on axis) for up to $10 \mathrm{~s}$. This is achieved by the combination of the SAIE high rate performance and a SA event buffer $\left(10^{6}\right.$ events $)$ on the PDHU.

A more general and detailed description of the AGILE PDHU may be found in Argan et al. 2004.

\subsection{SuperAGILE Burst Search and onboard Imaging}

Given its field of view and sensitivity, SA has a good chance to detect and localize cosmic gamma-ray bursts (GRBs), and this is indeed one of the most important scientific goals of the experiment, due to its combination with the simultaneous observation in the GeV energy range. Thus, a particular care was devoted to this issue during the design of the entire AGILE payload. In particular, SuperAGILE was equipped with an onboard trigger logic, able to promptly acknowledge the detection of a GRB, start an imaging process to localize its coordinates, activate a fast-communication channel based on the 
ORBCOMM data transmission network 3 to distribute the GRB coordinates to any ground- or space-based observatory almost in real-time.

However, the extremely tight limitations in the onboard processing capability allocated to this task imposed to make the burst search and imaging procedure as simple as possible.

\subsubsection{Burst Trigger Logic}

The burst trigger algorithm continuously and simultaneously compares the count rate of each detector, in two energy ranges, on 6 Short Integration Times (SITs: 1, 16, 64, 256, 1024 and $8192 \mathrm{~ms}$ ) with a background rate evaluated over 7 possible Background Evaluation Times (BETs: 8, 16, 32, 65, 131, $262 \mathrm{~s}$ ), to be associated to each SIT by telecommand. Also the type of comparison can be selected by telecommand between statistical (i.e., the excess or total counts are compared with the standard deviation of the background count rate, as evaluated by the average in the SIT) or static (i.e., the excess counts are compared to a fixed threshold in counts). The trigger criteria are independently continuously verified for the 8 ratemeters (4 detectors, 2 energy ranges) on the 6 SITs. Any possible configuration "triggered/not-triggered" of this 48-element pattern is then defined in a look-up table to originate or not a SA Burst Trigger. Once a SA Burst Trigger is declared in the PDHU, the following actions take place (when enabled):

- the SA imaging procedure is started (see next section);

- the download in the telemetry of the event-by-event data for the Minicalorimeter (Labanti et al. 2006) is activated over a time span dependent on a ded-

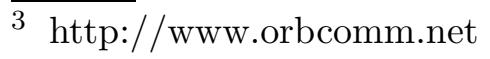


icated algorithm.

While processing a SA Burst Trigger, the burst search is inhibited for a fixed time interval, programmable from ground between 0 and $65535 \mathrm{~s}$.

\subsubsection{Burst Imaging and Coordinate Transmission}

The goal of triggering on GRBs is related to the ability to promptly localize them with arcmin accuracy in the sky. To this purpose, we devoted cyclic buffers in the PDHU to accumulate detector images for each of the $4 \mathrm{SA}$ detectors. The integration time is programmable, typically we expect to set it at $\sim 60 \mathrm{~s}$. Two images are accumulated for each detector at any time: a stored background image and a running foreground image.

The events used to build the images are the SA Good Events. Due to the varying attitude of the spacecraft (predicted as $0.1^{\circ} / \mathrm{s}$ inside a cone with $1^{\circ}$ aperture), a realtime attitude correction is needed in order to recover the blurring of the images due to the attitude variations. In practice, in the onboard procedure of building up the images, for each detected photon instead of incrementing the image pixel corresponding to the hit strip, another one is incremented whose coordinate is derived on the basis of the projection of the attitude error at the time of the photon on that specific direction. The attitude information is provided in realtime every $0.1 \mathrm{~s}$ by the AGILE Star Sensors with arcmin accuracy.

In principle, any region in the sky would require a specific correction but this implies an iterative procedure that cannot be performed onboard due to the very limited computing resources allocable to this task. We thus decided to 
process all the photons in the field with an average correction corresponding to the a-priori most probable incoming direction of the burst (obtained by the analysis of throughput, $\mathrm{cm}^{2} \times$ solid angle), that is $20^{\circ}$ off-axis. The effect of such an approximate correction will be a not perfect recovery of the attitude blurring. Since the high frequency attitude variations are expected to be symmetric, the net effect for a short timescale image is expected to be a loss in imaging sensitivity (that is, the peak in the sky image for a point source will be broader and thus dimmer) but without significantly affecting the peak position determination (see also $\S 13.2$ ). Long integrations, in general, are not expected to be used onboard.

When the SA Burst Trigger arrives, the background images of all the four SA DUs are "frozen", the foreground images reset, and the accumulation of new foreground (burst) images starts. Indeed, the background images are always kept $\sim 16$-20 seconds "in the past", in order to avoid to include the early burst photons in the background images. The end of accumulation of the burst image is reached when the signal/noise ratio of the image is evaluated to be large enough to allow a reliable coordinate determination. This is obtained by means of an algorithm, taking as input the pre-burst background count rate, the realtime counts and the desired statistical accuracy. When the stop command arrives, the realtime accumulation ends, the previously buffered pretrigger burst photons are added to the burst image, based on the timescale (SIT) of the burst trigger.

The next step is the subtraction of the background image from the image including the burst, after proper re-scaling of the integration time (so that persistent sources are removed from the net image), and the deconvolution of the net burst image with the mask code by means of Fast Fourier Transform. 
The resulting sky images are then co-added for the homologous detectors and the source peak is identified in each of the two coordinates.

A further validation step is foreseen based on the statistical significance of the peak in the sky images. If unambiguous coordinates are identified a special burst telemetry packet is generated. A subset of these information (trigger, SIT and image duration times, coordinates, pointing, count rates and other information relevant to the burst) are transmitted to the AGILE Bus for being sent through the ORBCOMM transponder to the relevant network for its transmission to the ground as an e-mail message. The typical delivery time of these messages is anticipated to be less than 2 minutes for more than $70 \%$ of the messages, being dependent on the relative instantaneous position of the AGILE and ORBCOMM satellites.

\section{SuperAGILE Data}

SuperAGILE was designed to transmit photon-by-photon data. The data type transmitted by the experiment to the AGILE telemetry can be summarized as follows:

(1) Good Event (GE): each of the two SAIEs drives 8 daisy chains and it is able to handle simultaneous events (that is, events on different daisy chains detected within $2 \mu \mathrm{s})$. For each event, SA transmits into telemetry the event type, its 13-bit strip address (reconstructed onboard through an address look-up table), 6-bit amplitude, 12-bit differential time (the time from the previous event, with $2 \mu$ s resolution);

(2) Absolute Time (ABT): the 32-bit Absolute Time events are generated 
by each SAIE every 99 events, transmitted as a pair of events; they are needed to convert the differential time to the On Board Time (OBT, in turn related to the UTC by means of the synchronization with the GPS performed every $1 \mathrm{~s})$;

(3) Dummy Events (DE): generated by the SAIE in case no real events are recorded within the maximum range of the differential time, $8.192 \mathrm{~ms}$; they report the differential time value;

(4) Calibration Events (CAL): the events generated during the Electrical Calibration and Threshold Scan procedures; these are sequential and not identified with a time stamp;

(5) Analog Housekeepings (AHK): included in the general AGILE HK telemetry packet, they report temperatures, voltages, currents and the experiment configuration parameters;

(6) Digital Housekeepings (DHK): included in the general AGILE HK telemetry packet, they report the 16-s hardware rates of accepted and rejected events;

(7) Scientific Ratemeters: described in 7, they are included in the general AGILE HK telemetry packet;

(8) Burst Alert: a telemetry packet generated when an onboard SA Burst Trigger is detected and the corresponding function is enabled; it includes information about the event (timescale, counts, coordinates, ...);

(9) Burst Imaging: a telemetry packet generated when an onboard trigger is detected and the corresponding function is enabled; it includes the images integrated onboard from which the burst coordinates were derived;

(10) Imaging Raw: a telemetry packet generated when the event-by-event transmission is inhibited (a solution to apply in case of a desired smaller telemetry allocation to SA); it includes continuous onboard images for 
each detector with integration time to be set from ground in the range 4 - 128 seconds.

The data types listed in (1) to (4) are indeed transmitted in the same telemetry packets, and they need to be separated by the ground software based on their identification flags.

\section{On-ground Tests and Performance}

The SA experiment was tested at any step of its integration. Specific test equipments (TEs) were designed and built to control and read-out the SAFEE and the SAIE boards (TE-SAFEE and TE-SAIE, respectively, see Pacciani et al. 2007 for a detailed description). These equipments allowed to test efficiently the SA detectors both stand-alone and integrated with the SAIE, by means of electronic pulses and radioactive sources. When integrated inside the AGILE payload the tests were performed using the specific Electrical Ground Support Equipment developed for the AGILE payload and satellite (e.g., Trifoglio et al. 2004). In this section we overview the results of the SA functional tests and the preliminary results of the ground calibrations carried out at experiment level and after the integration with the spacecraft.

\subsection{Functional Tests Results}

Functional tests were used to optimize the SA performance during the development phase, and to monitor the SA functionality and performance across the integration and environmental tests. These tests can be performed injecting an electrical stimulus (electrical calibration, threshold scan), or X-ray pho- 
tons from radioactive sources or integrating the environmental background. The electrical calibration is the most used test, because it can be performed anywhere in a relatively short time, without requesting any external source, and it tests all the channels individually. The results of an electrical calibration can be used to test the noise level, the gain, and the analog response of the address signals. Since the gain and the noise are drastically different if the detector strip is connected or not to the ASIC input channel (e.g., because of a broken wire bonding) this test can be used as an integrity check, although the detection of X-rays is the ultimate proof for it. The address signals, in turn, allow to study the address conversion look-up table as a function of instrument (e.g., power supplies) or environmental parameters (e.g., temperature).

\subsubsection{Electrical calibration}

Figures ?? and ?? show the synthetic results of an electrical calibration of SA obtained in March 2007 during the latest functional tests just before the shipping of the spacecraft to the launch base in India. Figure ?? reports the value of the gain of each individual strip, in ADC units per fC, as measured by a linear fit to the response of each channel to 1024 pulses with 4 different amplitudes (here the amplitude corresponds to $1.35,1.96,2.45,3.06 \mathrm{fC}$, that is $30.5,44.3,55.4$ and $69.2 \mathrm{keV}$ nominal). Figure ??, instead, shows the energy resolution (Full Width at Half Maximum, in units of $\mathrm{keV}$ ) derived for each strip from the same set of data. The reported value is the average of the values obtained for each of the 4 amplitudes, converted into keV by using the measured gain individually for each strip.

The gain is relatively uniform for all the channels, in the range 200-300 
ADC/fC. Also the energy resolution is around $8 \mathrm{keV}$ for most channels. Only the detection unit D2 has a significant fraction of channels with a lower gain and a higher level of noise. For D2, in those ASICs interfacing the edges of the two ladders, this is due to an accidental damage occurred during the ground calibrations, likely due to an electrostatic discharge. This event caused approximately 200 channels, distributed over 4 chips, to be permanently disabled, while the others channels of this unit survived but some of them with a higher level of noise. In fact, the same event provoked also a permanent increase in the dark current of the relevant detector, now $\sim 3.5 \mu \mathrm{A}$, compared to the typical $\sim 1 \mu \mathrm{A}$ of the other three detectors, at room temperature. From these plots it is also possible to identify the XA channels not connected to the corresponding detector strip because they show a higher gain and a better energy resolution, due to their smaller capacitive load.

\subsubsection{Threshold Scan}

This type of test is aimed to establish the relation between the discriminator threshold voltage (in $\mathrm{mV}$ ) and the charge (in $\mathrm{fC}$ ) for each channel of each XA ASIC chip. It consists of a sequence of electrical calibrations, scanning different values of the discriminator threshold. An operational definition of threshold is given as the discriminator reference voltage for which for a given charge injected to the test input of the ASIC the number of counts detected corresponds to half of the number of injected pulses. The outcome of this procedure thus allows to obtain an energy calibration of the setting of the analog thresholds, for a given experimental set-up. This procedure is significantly time consuming, as it consists of an electrical calibration repeated as many times as the number of threshold steps, typically 25. It also produces a 
huge amount of telemetry: with 4 amplitudes, 128 pulses per amplitude and 25 threshold steps it requires $\sim 1.8$ Gbit of telemetry, corresponding to $\sim 12$ orbits of standard operation. This implies that this procedure will need to be considered for use in orbit only in exceptional cases, at least in its complete form.

In Figure ?? we show the threshold of each channel, in $\mathrm{keV}$, for a given value of threshold DAC setting, that is the same nominal voltage reference. The commandability allows to set different voltage settings for each daisy chain (with a step corresponding to $\sim 1.5 \mathrm{keV}$ ). To decrease the dispersion between channels belonging to the same daisy chain, a 3-bit DAC setting is available for each of the 384 channels, and can be set through its shift register (Reg-In). The threshold equalization using these internal DACs is extremely complex and cumbersome, because they induce correlated variations to all channels of the daisy chain. In addition, the threshold setting is significantly temperature dependent. The analog threshold equalization was devoted a specific one-week test run that allowed to reach a threshold configuration dispersion of $\sim 0.8 \mathrm{keV}$ (rms, over the entire SA experiment), compared to the intrinsic $\sim 2.5 \mathrm{keV}$ for a non equalized set of thresholds. The technique and procedure used, as well as the details of the results, will be extensively discussed elsewhere (L. Pacciani et al., 2007b). The analog threshold configuration shown in Figure ?? is the one loaded into the SA front-end electronics at the time of the launch.

\subsubsection{Environmental Background}

The electrical calibration provides a measurement of the electronic noise through the measurement of the energy resolution in response to mono-energetic pulses. 
However, the energy resolution is only indicative for the discrimination threshold, that requires dedicated measurements. In particular, setting the experiment in photon acquisition mode, with a reasonable threshold setting, provides an energy spectrum whose components are expected to be only the environmental background and the high-amplitude tail of the electronic noise. The former can be easily distinguished by its flat spectrum, thus allowing to estimate the count rate of the electronic noise (expected to peak at low amplitudes) as a function of the threshold voltage. Fig. ?? shows a representative energy spectrum of the background, obtained with unit D3 and a nominal threshold of $17 \mathrm{keV}$, with the fine thresholds setting in its flight configuration. The low energy peak due to the electronic noise and the flat environmental background components are clearly distinguished. The broad peak at $\sim 95 \mathrm{keV}$ is due to the saturations of the individual channels, broadened by the gain equalization of the $\sim 1500$ active detector strips. The setting of the analog thresholds in orbit will be defined on the basis of the detected level of the electronic noise (highly dependent on the temperature of the front end electronics) and the allowed telemetry load.

\subsubsection{Radioactive X-ray sources}

The Electrical Calibration procedure allows to calibrate the response of the signal processing chain, from the charge preamplifiers inside the ASICs to the digital processing of the event. However, the photon detection capabilities need to be tested using X-ray photons. This allows to avoid the uncertainties about the test capacitor in the ASIC, that converts the test voltage pulse into a test charge, and the noise of the test circuitry itself (it has to be noted that the charge equivalent to $10 \mathrm{keV}$ in $\mathrm{SA}$ is obtained with a test pulse as 
low as $0.88 \mathrm{mV}$ ). In Fig. ?? and Fig. ?? we show the energy spectrum of the $\mathrm{Cd}^{109}$ (main line at $22 \mathrm{keV}$ ) and $\mathrm{I}^{125}$ (line complex at $27-32 \mathrm{keV}$ ) radioactive sources obtained with the D0 and D1 units, respectively. From such type of measurements the linearity curve for each of the four SA detection units can be obtained. Indeed, being the analog chains all independent, one should derive a linearity curve for each channel, but this conflicts with the counting statistics that can be achieved with reasonable experimental set-ups and integration times. As shown in Del Monte et al. (2007) the channel-energy conversion obtained with X-ray photons test is linear up to $120 \mathrm{keV}$, well outside the nominal SA energy range. The value of the slope coefficient is $3 \%$ larger than the corresponding one obtained with the electrical calibration. This is most likely due to the average difference between the real test capacitors of the ASIC channels with respect to their nominal $0.5 \mathrm{pF}$ value.

From the test runs with radioactive X-ray sources we can also derive an estimate of the energy resolution, independent from the electrical test pulse properties. For example, for the lines of the $\mathrm{I}^{125}$ complex, dominated by the $\sim 27 \mathrm{keV}$ line, we derive a value of $(8.3 \pm 0.1) \mathrm{keV},(8.3 \pm 0.1) \mathrm{keV},(8.4 \pm 0.1)$

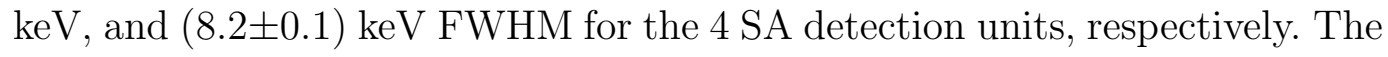
consistency of these values with those obtained with the electrical calibration procedure (\$ 9.1.1) implies that, with respect to its noise performance, the latter measurement is not significantly affected by the layout of the onboard test circuit, including its section internal to the ASIC itself, and can be taken as a good indicator of the instrument performance in orbit, where monochromatic lines from radioactive sources are no longer available. 


\section{On-ground Calibrations}

The SA experiment was calibrated on ground in three subsequent steps: at detection plane level (before the integration with the mask and collimator system and with the SAIEs) in IASF Rome on March 2005; at experiment level (with the experiment fully integrated but before the integration with the AGILE payload) in IASF Rome on August 2005; at satellite level at the Carlo Gavazzi Space facilities in Tortona (Alessandria, Italy) on January 2007. The details of the 2005 calibrations at subsystem level are given in Evangelista et

al. 2006, and Donnarumma et al. 2006. An extensive and detailed analysis of the SA performance on ground, as derived by the final ground calibrations, will be published in a forthcoming paper. Here we summarize the main results.

\subsection{Calibrations at Experiment Level}

\subsubsection{Calibration of Detection Units}

The detection plane was studied on March 2005 by means of standard radioactive sources (at 22, 32, 59 and $122 \mathrm{keV}$ ), and a quasi-parallel beam obtained by collimating an X-ray tube to a rectangular beam with the short side narrower than the detector strip width (approximately $100 \mu \mathrm{m}$ by $1.0 \mathrm{~mm} / 1.8$ $\mathrm{mm}$ at the Silicon) and with a divergence of approximately 0.7 arcmin. The spectrum was a bremsstrahalung continuum, peaking around $35 \mathrm{keV}$. Using these two set-up systems we studied the detector linearity, energy response, the dead time and the uniformity of the detection area. At the time of these measurements the SAIE boards were not fully completed and tested yet, thus the tests described in this section were carried out directly interfacing the 
SAFEE boards to their Test Equipment (see Pacciani et al. 2007 for details).

The linearity and energy resolution issues were discussed in $\S 9.1 .4$ and in the cited reference papers. The study of the detection uniformity showed a loss of about $4 \%$ of the counts at the border region between pairs of adjacent strips, likely caused by charge loss due to border effects of the electric field. Similarly, we found that the edge strips of the tiles have a larger efficiency, due to the fact that the guard ring, here limiting the strip, is farer than the strip pitch elsewhere in the detector. The increase in active area of the edge strips of each Si tile is $\sim 10 \%$, but in this case it is almost negligible, due to the fact that it applies to only 4 strip per detector. In any case, the above effects will be included in the instrument response matrix.

The instrument response to high count rates was tested with the X-ray beam, sending up to 1500 counts/s on each strip, individually. For one single strip this count rate corresponds to a source more than $10^{6}$ brighter than the Crab Nebula. Considering one daisy chain, instead, such a rate corresponds to $\sim 400$ times the Crab. Despite such a high rate, the detector response showed no distortions and the power consumption did not change appreciably. The estimated dead time per daisy chain was consistent with that derived from the timing as set by the relevant parameters (TrigDelBias, TrigWBias, ResWBias) for that acquisition, approximately $240 \mu \mathrm{s}$. In the flight configuration set-up this value (the dead time per daisy chain) was lowered down to $80 \mu$ s (see $\S$ 3.2).

Further details on the set-up and the measurements may be found in Evangelista et al. (2006). 


\subsubsection{Imaging Response at Experiment level: parallel beam}

The same collimated X-ray tube apparatus was used to test the imaging response of the experiment, after its definitive integration and alignment. The set-up included the SAIE boards and the measurements were therefore carried out using the SAIE Test Equipment (see Pacciani et al. 2007 for details).

The goal of this measurement was to obtain information about the point spread function (PSF) of the experiment, thanks the small divergence of the impinging beam thus providing a good approximation of a parallel beam. However, a recognized significant limitation to this measurement is related to the small extension $(\sim 1.8 \mathrm{~mm})$ of the beam along the mask strip. This implies that the real mask is sampled only in that specific section, and any local defect will have a much larger impact on the response than it will be in reality, when the complete mask will be sampled and the imperfections averaged out. Since a complete scan of the mask surface was made impossible by the schedule constraints, this measurement was complemented by a calibration of the experiment imaging response to a global illumination by omnidirectional sources (see next section), although in this case the beam divergence must be corrected for and the source extension subtracted. Again due to time constraints, the calibration with the X-ray tube was carried out on axis on the four detectors, and at $10^{\circ}$ and $20^{\circ}$ off axis for one detector only.

In Fig. ?? we show the deconvolved images of the (approximately) on-axis beam, as observed by the four detectors in the energy range $27-45 \mathrm{keV}$. This energy selection was applied because at the time of this measurement (August 2005) the analog threshold equalization had not been carried out yet (see $\S$

9.1.2) and the non-uniformity of efficiency among the different channels, due to 
the their intrinsic analog threshold dispersion, would have artificially affected the image reconstruction.

The 4 images show the source counts mostly distributed over three sky bins, as expected by the design of SuperAGILE. Indeed, the distribution of counts over the reconstructed sky pixels is a complex issue (see Evangelista et al. 2006 for an extensive discussion). Due to the discrete pixels of the detector, for an ideal mask and detector, the counts to be expected in the three pixels on the basis of the ideal PSF depend on the exact angular position of the source, that is the relative phase between the mask element and the detector element, with a period of one detector pixel ( 3 arcmin). At phase 0 , the counts are distributed according to the proportion 0.5:1.0:0.5 (meaning that the sum over the 3 bins is twice the source counts). In the "worst case", they will be distributed over 4 pixels, according to the ratio 0.25:0.75:0.75:0.25. Intermediate phases provide intermediate distributions of counts over 3 or 4 pixels. Unfortunately, our setup did not allow a measurement of the beam inclination accurate enough, thus the phase remains an unknown for all the measurements, different for each one, being the Detection Plane rotated at every measurement and having each of the four detectors, at this level of accuracy, its own independent optical axis, related to the fine details of its construction. Since the relative phase can only result in the distortion of the symmetry of counts distribution, the almost perfect symmetry of image from D2 and D3 implies that the beam was very close to phase 0 in these cases, and this allows to confirm that for these two systems (detector plus mask) the response is very close to the ideal one. The most symmetric case, D3, shows significant counts over 5 contiguous pixels, with intensity with respect to the total source counts according to 0.04:0.50:0.94:0.49:0.04. The difference between this proportion and the ideal 
one is understood as due to the physical effects (e.g., mask transparency at high energies, finite thickness of the mask, Compton scattering of photons on the mask, ...) and to the distortions of the specific section of the real mask sampled by this measurement. A deeper analysis of these calibration data is under way in order to understand in greater details the instrument imaging response function. The results will be reported in a future publication.

The same measurement was carried out at two off-axis angles for unit D0. In Fig. ?? we show the relevant images together with the on-axis measurement on the same unit, for comparison. These plots allow to appreciate how the SA PSF is only weakly dependent on the source position in the FOV, on at least a large portion of its effective $2 \mathrm{x} 1 \mathrm{D}$ FOV $\left(\sim 60^{\circ} \times 60^{\circ}\right.$, at zero response).

Indeed, some distortions in the images are expected when the source is offaxis, increasing in amplitude as the off-axis angle increases. This is due to the incomplete sampling of the mask code. In fact, our selected mask code is fully balanced only for on-axis sources, while the rest of the field is partially coded and the images show the effect of the coding noise. In Figure ?? we show these distortions in the above measurements by showing the SA image of the full sky, in place of the zoom-in on the source. As discussed in $\S$ 4.1. these distortions are largely compensated when the homologous DUs are used to image the same source on the same coordinate, thanks to the mask/anti-mask imaging technique. Alternatively, balancing techniques can be applied also for individual DU detections. On the other hand, the same figure allows to appreciate the nice flat-lobe response of the SA optical system to on-axis sources. At large off-axis angles a contribution to the peak broadening is expected also as an effect of the inclined penetration of photons in the silicon. 


\subsubsection{Imaging Response at Experiment level: divergent beam}

As discussed in the previous section, due to set-up and time limitations, the parallel beam illumination of SA could provide the experiment imaging response only for a small $(\sim 2 \mathrm{~mm})$ section of the mask. To the purpose of calibrating the average instrument response, the only reasonable alternative was to use omnidirectional radioactive $\mathrm{X}$-ray sources. To this purpose we developed a procedure (see Donnarumma et al. 2006 for details) to correct for the beam divergence and the physical source extension. With such procedure, we were able to derive properties of the SA response to an infinite distance source using finite distance instead. Of course, the procedure is increasingly accurate as the source distance increases. On the other hand, this leads to larger integration times, and a trade-off needs to be found. The final aim of these measurements at experiment level was to test and calibrate the procedure in view of the final experiment calibrations (see next section), as well as deriving some early information about some experiment parameters and the imaging response. In fact, during the August 2005 calibration campaign only a short time could be allocated, and the set of measurements is relatively poor thereof.

The distance between the source and the Detection Plane was set at $\sim 200 \mathrm{~cm}$, leading to a beam divergence of $\sim 6^{\circ}$ within one detector. The measurements were carried out with a $\mathrm{Cd}^{109}$ radioactive source (main line at $22 \mathrm{keV}$ ) at $0^{\circ}$, $10^{\circ}, 20^{\circ}$ and $30^{\circ}$ off-axis for D0, and with a $\mathrm{Cs}^{137}$ source (line of interest, 32 $\mathrm{keV}$ ) on axis only. The exact position of the radioactive sources with respect to SA was measured by means of a laser tracker, having mounted optical reference targets on the source holder and optical cubes on SA. Since the reconstruction procedure for finite-distance source imaging is sensitive to the ratio between 
the mask-detector distance and the source-mask distance, the optimization of the image reconstruction allowed to derive from multiple measurements an independent estimate of the mask-detector distance. The derived value of $(142.44 \pm 0.10) \mathrm{mm}$ for unit D0 (for which the full set of measurements is available) is consistent with both the experiment mechanical design and the measurement derived by the detailed micrometric precision mapping of the experiment carried out with the metrology machine during the experiment integration.

In Figure ?? we show a sample of the reconstructed source images obtained with D0 during this calibration session. (For a more complete and detailed description we refer the reader to Donnarumma et al. 2006). Although these images are not yet corrected for the source extension (approximately 8 arcmin) they confirm the expected PSF up to $30^{\circ}$ off-axis. The detailed determination of the PSF parameters from these measurements requires an accurate mapping of the space distribution of the radionuclide, partly responsible for the distribution of counts. This measurement is currently planned but not yet carried out.

\section{Ground Calibrations at Satellite Level}

The final SA ground calibrations were carried out for one week on January 2007, when the experiment was onboard the fully integrated satellite. The set-up was based on the experience of the August 2005 calibrations, with radioactive X-ray sources located at $\sim 200 \mathrm{~cm}$ from the SA mask. The source support was studied and manufactured in order to avoid some systematic uncertainties on the radionuclide location revealed during the 2005 calibrations, 
and it was mounted on a movable structure, guaranteeing at the same time the satellite safety, the source mobility at a distance of $\sim 4 \mathrm{~m}$ from ground (the satellite was in vertical position, placing $\mathrm{SA} \sim 2 \mathrm{~m}$ above the floor) and the required micrometric stability. The micrometric position of the source was targeted with a laser tracker also in this campaign, with respect to reference points on the spacecraft structure, and to the reference optical cubes aligned to the AGILE star sensors.

For this calibration campaign 3 radioactive sources were used: $\mathrm{Cd}^{109}$ (main line at $22 \mathrm{keV}$ ), $\mathrm{I}^{125}$ (line complex at 27-32 keV) and $\mathrm{Am}^{241}$ (main line at 59.5 $\mathrm{keV}$, plus line complex at 14-26 keV). The largest sampling was carried out using the brightest source, $\mathrm{Cd}^{109}$. In order to save time, the same measurement was used for the 4 DUs, implying that slightly different off-axis positions were sampled for each DUs. In particular, we took as a reference D0 and built a "cross" in its field of view, from $-50^{\circ}$ to $+50^{\circ}$ off-axis in both coding and non-coding directions, step $10^{\circ}$. The central square included in $-20^{\circ}-+20^{\circ}$ in both directions was also filled with $10^{\circ}$ steps. Finally, we took measurements at $\left(-30^{\circ},-30^{\circ}\right),\left(-30^{\circ},+30^{\circ}\right),\left(+30^{\circ},-30^{\circ}\right)$ and $\left(+30^{\circ},+30^{\circ}\right)$, for a total of $55 \mathrm{FOV}$ positions sampled with the $\mathrm{Cd}^{109}$. The other two sources were instead used to sample fewer position near the center of the FOV, for a total of 12 measurements with the $\mathrm{I}^{125}$ source and 3 measurements with the very weak $\mathrm{Am}^{241}$, for which the distance had to be decreased down to $\sim 100 \mathrm{~cm}$. As discussed above, for the other 3 SA DUs the off-axis angles were slightly different, due to the finite distance of the source, the relative off-set being in the range of $5^{\circ}-10^{\circ}$, depending on the source position.

In Figure ?? we show a sample of the zoomed source images for one of the SA DUs. The analysis of the complete data set collected during these recent 
calibrations is still under way. A detailed report will be given in a future publication. Here we remind the goals we aim to, for some of which preliminary results are already being obtained, for each of the 4 DUs: determination of the PSF as a function of energy and position in the FOV (this will require also the subtraction of the source extension, after its measurement), calibration of the relation between the detector pixel and the exact off-axis angle, response as a function of energy, map of obscuring structures in the FOV (mostly the support structure of the anticoincidence system and some harness), estimation of the accuracy of localizing single point sources.

During the same calibration campaign some time was devoted to study the effect of a source location outside the FOV, i.e., searching for leaking directions in the experiment shielding to the diffuse X-ray background. A few leaking or partially leaking directions were found, but none of them seriously affecting the experiment performance. They are being accounted for in the experiment response function.

\section{The Ground Software: SuperAGILE Data Analysis System (SADAS)}

Despite the very limited resources, SA is a photon-by-photon experiment and requires a full data processing on ground. The SA Scientific Software was entirely developed at IASF Rome and it is planned to run autonomously at the AGILE Data Center (ADC) located at the ASI Science Data Center (ASDC) in Frascati for the standard analysis and at IASF Rome for the refined analysis, with human support. The source position and fluxes will be made publicly available by ASDC in the shortest time to the whole science community through the a dedicated SA web page. 
The SA data processing starts with the AGILE data transmission from the spacecraft to the Malindi ground station. From there, data are transmitted to the ASDC where the telemetry is pre-processed and archived. The preprocessing is carried out following the DISCOS system already used on previous astronomy missions (e.g., Gianotti et al. 2001) and transforms the raw telemetry data (Level 0) to a FITS standard format (Level 1). The subsequent processing is in charge to the SA Pipeline, that will run automatically at every ground contact (i.e., nominally every 100 minutes). The basic conceptual steps of the pipeline are:

(1) Data Quality Filter;

(2) Separation of data types: events, calibration, absolute time, dummy;

(3) Creation of photon list;

(4) Attitude correction;

(5) Source extraction by an Iterative Removal of Sources procedure;

(6) Source identification.

Each of these steps is a complex procedure itself. We refer to Lazzarotto et al. (2007) for a detailed description of the SA ground software and pipeline processing. At different steps of pipeline, organized in 4 stages, data products will be archived. The final data products, in standard FITS formats, concerning the position and identification of the detected sources and their flux level will be transferred to the ASDC public archive for use by the general community.

The SA data processing will be carried at different temporal stages. At any contact (orbit) a prompt analysis of the data collected during the last available orbit will be carried out. This is aimed to obtain the fastest reaction to transient events. After the orbital processing is completed, the same data 
are added to those of the previous orbits looking at the same field, in order to identify weaker sources and improve the localization of unknown sources. Finally, the same data will then be reprocessed with the final auxiliary data (e.g., reconstructed attitude, ephemeris, ...) to provide their final scientific products.

\subsection{Real-time Alerts of X-ray transients}

As discussed in $\S$ 7.1, part of the onboard software was designed to trigger and autonomously localize fast hard X-ray transients, namely gamma-ray bursts and objects with similar phenomenology. The coordinates are sent in quasirealtime to ground through the ORBCOMM satellite link.

The SA ground segment thus includes a section that is devoted to process the ORBCOMM messages at their arrival, and to perform the refined data analysis after the satellite ground contact has made the relevant SA data available to the ASDC. The main features of this section are: validating the ORBCOMM message, validating the onboard trigger, confirming the reliability of the onboard localization, assign localization uncertainty, verify the coordinates against source catalogs, disseminate the coordinates of the hard X-ray transient to the general community through the standard communication channels (e.g., BACODINE, GCN, ATel, ...).

After the early processing, based on the data transmitted through the realtime message, the same trigger is validated and the trigger properties are refined by an on ground processing of the complete SA data set. In this phase the data of the other AGILE subsystems (GRID and MCAL) will be available as well, 
allowing to determine the high energy properties of the event in the $\sim \mathrm{MeV}$ and hundreds of $\mathrm{MeV}$ ranges. The results of these off-line analysis will also be distributed as quickly as possible to the widest community.

\section{Astrophysical Expectations}

SuperAGILE was designed as the hard X-ray monitor of the AGILE gamma ray imager, and needed to fit tight design constraints. However, considering the emission properties of the known gamma-ray sources and the SA expected sensitivity, the classes of known sources where the two instruments are really expected to work together are only a few (but SA is in the best position to extend this set to other classes of sources, especially variable ones). However, SA will also work as an autonomous instrument, taking advantage of its wide field of view and reasonable sensitivity. In this section we briefly review the expected performance of the experiment and outline the potential contribution of SA to the current scenario of the observational X-ray and gamma-ray astronomy.

\subsection{Effective Area and Sensitivity}

The SuperAGILE response was computed by means of analytical calculations and Monte Carlo simulations, based on a model describing the geometry and mass of the experiment in great detail. In Fig. ?? we show the effective area, as a function of energy and angular position of the source, as computed by means of analytical calculations. The peak of the effective area is at about 14 $\mathrm{keV}$, just below the effective SA lower energy threshold (determined mostly 
by the electronic noise), and it is due to the absorption of the intervening material (mainly the plastic anticoincidence and its support structure) at low energy, and the Silicon efficiency decrease at high energy. Due to the inclined penetration of radiation in Silicon, at higher energies the peak shifts as the off-axis angle increases, reaching $\sim 16 \mathrm{keV}$ at $50^{\circ}$.

Assuming a source with an energy spectrum similar to the Crab Nebula $\left(I(E) \sim E^{-2}\right)$ and simulating the SA response to the source and to the diffuse X-ray background, we can compute the expected sensitivity to a single source as a function of its location inside the field of view. With an integration time of 50,000 s (effectively, about one day of observation, for typical orbital constraints in the AGILE low-earth orbit) the results of our simulations are shown in Fig. ??, in terms of sensitivity for two homologous detectors, in the energy range $15-45 \mathrm{keV}$, in units of mCrab ( 1 mCrab corresponds to $4 \times 10^{-4}$ photon $\mathrm{cm}^{-2} \mathrm{~s}^{-1}$ or $1.5 \times 10^{-11} \mathrm{erg}^{-2} \mathrm{sm}^{-1}$ in this energy range), as well as for the combination of the 4 detectors, imposing an independent detection on each coordinate. The SA sensitivity thus appears rather "flat" over the central $\sim 60^{\circ} \times 60^{\circ}$ of the field of view, going from $\sim 15 \mathrm{mCrab}$ in the central region to $\sim 50$ mCrab at $\sim 30^{\circ}$ off axis, making the simultaneous monitoring of large regions of the sky an achievable goal for the experiment.

\subsection{Point Source Location Accuracy}

The statistical point source location accuracy (PSLA) in coded mask experiments like SA depends on the ratio between the position resolution of the detector and the factor $f \times S N R$, where $f$ is the mask-detector distance and $S N R$ is the signal-to-noise ratio of the specific measurement (e.g., in 't Zand 
1992). Given the SA 3 arcmin on-axis pixel size, the PSLA for a $5 \sigma$ source should be already in the sub-arcmin region. At off-axis angles the SA pixel decreases in angular size (down to 1.5 arcmin at the edge of the FOV), but the effective area is far smaller.

We verified the above relation by taking a long on-axis measurement with the $22 \mathrm{keV}$ source collected during the ground calibration and dividing it in a large number of statistically independent measurements, with SNR $\sim 5$ and $\sim 10$. In Figure ?? we show the distribution of the barycenter of the reconstructed image in one SA DU. Despite the measurement suffered the limitations of our finite-distance calibrations (see $\S 10.1 .3$ and $\S 11$ ), the measured distributions show an rms of 0.8 and 0.4 arcmin, respectively, only slightly worse than the theoretical 0.6 and 0.3 arcmin limits.

The imaging procedure of SA in its flight operation includes the attitude correction, needed to recover from the large attitude variations expected in orbit, due to the bus attitude control system. In fact, the AGILE spacecraft is only able to stabilize the attitude within $1^{\circ}$ of the requested target, with variation as large as $0.1^{\circ} / \mathrm{s}$. In addition to this large attitude variation on short timescale, the AGILE bus requires the solar panels to be orthogonal to the Sun within $1^{\circ}$, implying a continuous drift of the pointing to keep this constraint satisfied. The star trackers will provide an attitude reconstruction with an accuracy of $\sim 1$ arcmin with a $10 \mathrm{~Hz}$ frequency. Thus the position of all the SA photons must be corrected to their original position, with the strong limitation of the one dimensional imaging and the discrete pixels with size corresponding to 3 arcminutes. Indeed, the SA PSLA will be most likely effectively limited by the systematics in the satellite attitude stability and reconstruction. In Fig. ?? we show an example of the results of the attitude reconstruction procedure 
developed by the SA team, applied to a simulated image blurred by the expected in-flight attitude variation. The result of the correction is expected to preserve the "ideal" (that is, with no attitude variation) signal-to-noise ratio to within $95 \%$.

What above concerns the on-ground processing of the SA data. However, as discussed in $\S$ 7.1.2, the onboard burst triggering and localization also requires a real-time attitude correction of the events. Since this correction is dependent on the source location in the FOV, it requires an iterative procedure that cannot be supported by the onboard computing power. As a consequence, an approximate correction is performed, using an average correction parameter that is exact only for $\sim 20^{\circ}$, the off-axis angle at which the experiment has its largest throughput. For any other source position in the FOV, the attitude correction will be non optimal, resulting in a widening of the source peak, and a consequent loss in the source image significance. The fourth panel in Fig. ?? shows the effect of such an approximation. This will affect the onboard localizations of gamma-ray bursts distributed in real time through the ORBCOMM link (see Sect. 17). A ground analysis of the same data, to be performed after the data downlink at the passage over the Malindi Ground Station will be able to optimize the attitude correction, and the data analysis in general, and distribute refined and more accurate coordinates of the detected transient. This will happen with a delay varying between few minutes and few hours, depending on the transient occurrence time with respect to the data download carried out every $\sim 100$ minutes at the passage over the ground station. 


\subsection{The Expected SuperAGILE View of the X-ray and Gamma-ray Sky}

The main SA science goal relates to the simultaneous monitoring of the gammaray sky observed by the AGILE primary instrument, the gamma ray imager. The classes of astrophysical sources known to emit in the energy band 30 $\mathrm{MeV}-50 \mathrm{GeV}$ to a level of intensity compatible with the AGILE sensitivity can be listed as: gamma-ray pulsars, supernova remnants, Active Galactic Nuclei (mostly Blazars), gamma-ray bursts and the unidentified EGRET sources. The known hard X-ray emission of the gamma-ray pulsars and supernova remnants is largely below the SA sensitivity level, except for the case of the Crab Nebula and Pulsar, and possibly the Vela Pulsar. The X-ray flux of blazars is typically near or below the sensitivity threshold of SA, but in some cases outbursts of these sources brings an hard X-ray emission detectable by SA (e.g., Mkn 501, Pian et al. 1998). Also the brightest members of other classes of AGNs (quasars, Seyfert 1 and 2 galaxies, radio galaxies), are expected to be detectable with SA. In a few cases these are known emitters of gamma-rays, as 3C 273 and 3C 279 (e.g., Hartman et al. 1999).

Concerning the unidentified EGRET sources, almost nothing is known about their nature. The quest for hard X-ray counterparts has been recently carried out by INTEGRAL in an energy range similar to the SA one. No obvious counterpart has been found, except possibly in a few cases (e.g., Di Cocco et al. 2004, Foschini et al. 2005). Therefore, several or most of the unidentified EGRET sources are unlikely to be persistent and bright hard X-ray sources. But they may well be transient emitters and the very large field of view of SA might become crucial in their observation, in this respect. 
The situation becomes far more favorable as it comes to the gamma-ray burst sources. The prompt emission of these objects is largely above the SA sensitivity limit, even on their typical time scales (from tenths to tens of seconds). Based on its FOV and sensitivity, SA is expected to detect and localize gamma-ray bursts at a rate of $\sim 1-2$ events/month. This expectation was the driver to equip the system with an autonomous triggering and localization system, and with the fast down-link of the event coordinates. Although the SA localizations will be just a few with respect to those currently obtained by the Swift mission (Gehrels et al. 2004), they will be uniquely characterized by the simultaneous observation in gamma-rays. The AGILE data combined with the multi-wavelength follow-up studies might then be able to provide a complete view of $\mathrm{GeV}$-emitting gamma ray bursts, including their distance, energetics and counterpart.

The SA operation as hard X-ray monitor of the AGILE gamma-ray imager is complemented by its "independent life", as a wide field X-ray experiment. The large field of view, high angular resolution and moderate sensitivity makes SA suitable for (quasi-)all-sky monitoring. The hard X-ray sky is highly variable and new sources are continuously discovered, especially in the class of compact objects. Monitoring of known sources provides crucial information for their long-term behavior and allows observations with sensitive telescopes to be carried out at proper times and source states. The most favorable candidates for SA are the galactic sources, and this is also favored by the expected AGILE pointing plan, foreseeing large part of the observing time to be spent on the Galactic Plane. As an example, in Fig. ?? we show a simulation of a one-day SA observation of the Galactic Center region (sources as described by the second INTEGRAL ISGRI catalog, Bird et al. 2006). The Monte Carlo 
simulation, including a full description of the SA geometry, takes into account the simultaneous contribution of all sources and of the diffuse X-ray background. The data processing of this simulation with the SA ground software includes the iterative removal of sources, IROS.

\section{Summary and Conclusions}

In this paper we described the design, manufacturing, integration and ground performance of the SuperAGILE experiment, the hard X-ray monitor of the AGILE gamma-ray space mission. The ground calibrations demonstrated the nominal performance of the experiment in the 18-45 keV energy range. Lower energy thresholds may be achieved in orbit if the experiment temperature will be lower than the one at which it was operated during tests and calibration (between 35 and 40 degrees at the front-end). The measurement of the point spread function confirmed the expected three-bin triangular shape, with a 6 arcmin full width at half maximum, and a point source location accuracy that will likely be limited to $\sim 1$ arcmin by the spacecraft attitude reconstruction. The on-axis sensitivity is expected in the range of $\sim 15$ mCrab for a one-day exposure on an extragalactic field.

SuperAGILE was launched on $23^{r d}$ April 2007 in an equatorial orbit $(540 \mathrm{~km}$ altitude, $2.4^{\circ}$ inclination). The initial operations in orbit showed a nominal performance. The check-out phase will continue over the first two months and will be followed by a two-month science verification phase. The in flight performance and observational results will be subject of future papers. 


\section{Acknowledgments}

AGILE is a mission of the Italian Space Agency, with co-participation of INAF (Istituto Nazionale di Astrofisica) and INFN (Istituto Nazionale di Fisica Nucleare).

Along the ten years of the SuperAGILE design, development, manufacturing, integration and testing a number of people contributed from the involved companies of from research institutes to this work at any level, administrative, technical or scientific. We are pleased to acknowledge their individual contributions, without which the SuperAGILE project would hardly have been possible: the AGILE Co-Principal Investigator G. Barbiellini (INFN - Sez. Trieste), L. Barbanera, A. Bartocci, G. De Paris, C. Pala, G. Sabatino, B. Schena, O. Uberti (INAF/IASF, Rome), A. Morbidini (INAF/IFSI, Rome), L. Bettinali, A. Lo Bue, P. Rossi, L. Semeraro (ENEA, Frascati), A. Generosi, V. Rossi-Albertini, E. Verona (CNR, Rome-Tor Vergata), A. Russo (CNR, Naples), M. Conte, G. De Paris, L. Soli, A. Zambra, A. Trois (current and past AGILE System Team), S. Mereghetti, S. Vercellone (INAF/IASF, Milan), A. Bulgarelli, F. Fuschino, F. Gianotti, M. Trifoglio (INAF/IASF, Bologna), F. Longo, C. Pontoni, M. Prest, E. Rotta, E. Vallazza (INFN - Sez. Trieste), P. Bresciani, N. Kocjancic, D. Rossi, R. Starec, R. Terpin (MIPOT, Cormons), Z. Durna, J. Vincenz (ILFA Gmbh, Frankfurt), S. Mikkelsen, E. Nygaard, A. Suleyman, B. Sundal, K. Yoshioka (IDE AS, Oslo), L. Acquaroli, M. Angelucci, M. Angelucci, F. Antichi, G. Babini, M. Calabrese, B. Morelli, G. Pedrazzoli, U. Ricciardi, S. Sideri (Oerlikon Contraves Italia), E. Artina, P. Bastia, A. Bonati, G. Cafagna, L. Maltecca, G. Marseguerra, F. Monzani, L. Nicolini, M. Patane', R. Pavesi, P. Radaelli, F. Ticozzi, V. Vettorello, E. Zardetto 
(Thales-Alenia Space Italia, formerly Laben, Milan), S. Alia, G. Annoni, E.

Collavo, I. Ferrario, B. Garavelli, M. Giacomazzo, S. Legramandi, A. Longoni, C. Maini, P. Sabatini (Carlo Gavazzi Space, Milan), L.A. Antonelli, G. Fanari,

P. Giommi, C. Pittori, B. Preger, R. Primavera, P. Santolamazza, S. Stellato, F. Verrecchia (ASI Science Data Center).

\section{References}

[1] A. Argan, et al., Nuclear Science Symposium Conference Record, 2004 IEEE, Vol.1, Iss., 16-22 Oct. 2004 Pages: 371- 375.

[2] K. Bennett, Nucl. Phys. B (Proc. Suppl.) 14B (1990) 23.

[3] A.J. Bird, et al., Astrophys. J. 636 (2006) 765.

[4] P.M. Charalambous, A.J. Dean, J.B. Stephen, and N.G.S. Young, Appl. Opt. $23(1984) 4118$.

[5] E. Del Monte, et al., Nucl. Instr. and Meth. A 538 (2005) 465.

[6] E. Del Monte, et al., Nucl. Instr. and Meth. A 572 (2007) 708.

[7] G. Di Cocco, et al., Astron. Astrophys. 425 (2004) 89.

[8] I. Donnarumma, et al., 2007, in preparation.

[9] M. Feroci, et al., Proc. SPIE 5488 (2004) 165.

[10] C. Fichtel, et al., Astrophys. J. 198 (1975) 163.

[11] L. Foschini, et al., Astron. Astrophys. 433 (2005) 515.

[12] N. Gehrels, et al., Astrophys. J. 611 (2004) 1005.

[13] F. Gianotti, et al., ASP Conf. Ser. 238 (2001) 245. 
[14] I. Grenier, Proc. Texas in Tuscany. XXI Symposium on Relativistic Astrophysics, Florence, Italy, 9-13 December 2002, Eds.: R. Bandiera, R. Maiolino, F. Mannucci. Singapore: World Scientific Publishing, ISBN 981-238580-0, 2003, p. 397 (also astro-ph/0303498).

[15] R.C. Hartman, et al., Astrophys. J. Suppl. Ser. 123 (1999) 79.

[16] J.J.M. in 't Zand, Ph.D. Thesis, University of Utrecht, 1992, ISBN 90-393-04734.

[17] J.J.M. in 't Zand, J. Heise, and R. Jager, Astron. Astrophys. 288 (1994) 665.

[18] C. Labanti, et al., Proc. SPIE 6266 (2006) 62663Q.

[19] I. Lapshov, et al., 2007a, in preparation.

[20] I. Lapshov \& L. Pacciani, 2007b, in preparation.

[21] F. Lazzarotto, et al., 2007, in preparation.

[22] L. Pacciani, et al., Nucl. Instr. and Meth. A 574 (2007a) 330.

[23] L. Pacciani, et al., 2007b, in preparation.

[24] E. Pian, et al., Astrophys. J. 492 (1998) L17.

[25] M. Rapisarda, et al., 2004, SPIE 5488 (2004) 660.

[26] P. Soffitta, et al., Proc. SPIE 6266 (2006) 626631.

[27] M. Tavani, et al., Proc. SPIE 6266 (2006) 626603.

[28] M. Trifoglio, et al., Proc. SPIE 5488 (2004) 71.

\section{FIGURE CAPTIONS}

FIGURE 1: A sketch of the exploded SA structure. 
FIGURE 2: Photographs of one of the four flight units of the SAFEE boards, taken during the integration process in 2005. The photo on the left shows both the horizontal and vertical boards (here open), connected by the flexible section of the PCB. The photo on the right shows the integration process with the detector, with the vertical section closed in its aluminum box.

FIGURE 3: The flight unit of the SA Detection Plane after its mechanical integration in MIPOT.

FIGURE 4: The flight unit of the carbon-fiber/tungsten SA collimator. The bottom view allows to see the separation between collimator cells and the copper plates providing the electrical grounding of the structure.

FIGURE 5: A simulated SA exposure to a blank sky (i.e., no point sources), with the diffuse X-ray background partially blocked by the Earth. The top panel shows the detector images of the two homologous detectors. The mid panel shows the corresponding sky images, with the significant effect of the background inhomogeneity. The bottom panel is the sum of the two sky images, showing their compensation, due to the mask/anti-mask configuration of the two homologous detectors.

FIGURE 6: The flight unit of the SA mask-collimator system. The four individual coded masks are visible, as well as the sections at the outer edges where the sample strips were manufactured and stripped off for the quality control. The 3 optical cubes used for alignment purposes are also visible on the corners. 
FIGURE 7: SA in its flight configuration, integrated with the AGILE payload during the integration campaign in at the CGS facilities in Tortona.

FIGURE 8: Gain (ADC channel per fC) for all the 6144 individual channels of SuperAGILE, as measured on March 2007.

FIGURE 9: Energy resolution $(\mathrm{keV})$ for all the 6144 individual channels of SuperAGILE, as measured on March 2007.

FIGURE 10: Analog energy threshold setting (keV) for all the 6144 individual channels of SuperAGILE, as measured on December 2006, with the launch configuration setting.

FIGURE 11: Energy spectrum of the background in the D3 unit, with a nominal threshold setting at $17 \mathrm{keV}$. The energy units are given in Pulse Height Amplitude units, each one approximately corresponding to $1 \mathrm{keV}$.

FIGURE 12: Energy spectrum of a $\mathrm{Cd}^{109}$ radioactive source obtained with the SA unit D0, placing the source on axis at a distance of $\sim 200 \mathrm{~cm}$. The energy units are given in Pulse Height Amplitude units, each one approximately corresponding to $1 \mathrm{keV}$. 
FIGURE 13: Energy spectrum of a ${ }^{125}$ radioactive source obtained with the SA unit D0, placing the source on axis at a distance of $\sim 200 \mathrm{~cm}$. The energy units are given in Pulse Height Amplitude units, each one approximately corresponding to $1 \mathrm{keV}$.

FIGURE 14: Zoom-in of the images of the collimated beam from the X-ray tube, for an incidence angle near to the instrument optical axis and energy selected in the range $27-45 \mathrm{keV}$. Top left is unit D0, top right D1, bottom left D2 and bottom right D3. The angular size of each bin in these images is 3 arcmin.

FIGURE 15: Zoom-in of the images of the collimated beam obtained with unit D0 from the X-ray tube, for an incidence angle near to on-axis (left), $\sim 10^{\circ}$ (center) and $\sim 20^{\circ}$ (right) off axis and energy selected in the range $27-45 \mathrm{keV}$. The angular size of each bin in these images is 3 arcmin.

FIGURE 16: Same images as in Figure ??, but showing the full field of view, allowing to outline the image distortions due to the incomplete sampling of the mask code at off-axis locations. As above, incidence angle is near to on-axis (left), $\sim 10^{\circ}$ (center) and $\sim 20^{\circ}$ (right) off axis and energy is selected in the range $27-45 \mathrm{keV}$. The angular size of each bin in these images is 3 arcmin.

FIGURE 17: Reconstructed images of a $\mathrm{Cd}^{109}$ radioactive source $(22 \mathrm{keV})$ at $205 \mathrm{~cm}$ distance from SA, on axis (left) and $30^{\circ}$ off-axis (center). On the right, the image of a $\mathrm{Cs}^{137}$ source $(32 \mathrm{keV})$ on axis, at the same distance. The angular size of each bin in these images is 3 arcmin. 
FIGURE 18: Reconstructed images of a $\mathrm{Cd}^{109}$ radioactive source $(22 \mathrm{keV})$ at $\sim 200 \mathrm{~cm}$ distance from SA, for off-axis angles from $-40^{\circ}$ to $+50^{\circ}$, step $10^{\circ}$ (from top left to bottom right). The angular size of each bin in these images is 3 arcmin.

FIGURE 19: Effective area of one of the 4 SA Detection Units, derived by means of analytical calculations, as a function of energy, at different off-axis angles.

FIGURE 20: Left: Monte Carlo simulation of the 50 ks sensitivity of 2 SA Detection Units, as a function of the source position in the field of view, in the $15-45 \mathrm{keV}$ energy range. The sensitivity is expressed in terms of mCrab units. Right: combined sensitivity (bolean AND) of the 4 SA detectors. The given sensitivity in each point is the one relevant to the least sensitivity of the two pairs of detectors.

FIGURE 21: Estimation of the statistical SA Point Source Location Accuracy (here D0 unit) in terms of the distribution of the positions of the barycenter in the reconstructed image, in a sample of statistically independent measurements performed with a $22 \mathrm{keV}$ source at $200 \mathrm{~cm}$ distance, during the ground calibration. Panels on the left and rights show the measurement with a signalto-noise ratio of $\sim 5$ and $\sim 10$, respectively. 
FIGURE 22: Top: Sky image of the on-axis point-like source in ideal conditions with absolutely stable pointing. Second: Sky image of the same source without attitude correction. Source shape roughly matched the pointing error histogram for a given direction. In the case shown the pointing errors had a trend towards positive coordinates, thus making the uncorrected image to be centered in the wrong position. Third: The same as above, but after applying the on-ground attitude correction method. Note the decreasing of the coding noise in the image for angles above 30 degrees compared with the ideal case. Bottom: The same as above, but after applying the simplified on-board attitude correction method. Correction parameter was set for the best correction at 20 degrees off-axis in the field of view.

FIGURE 23: Left: Simulated SA observation of the Galactic Center region, as described by second INTEGRAL Catalog (Bird et al. 2006). Right: Sources identified in the same image by the SA IROS procedure after 10 iterations. 
Table 1

Main instrumental parameters of the SuperAGILE experiment.

\begin{tabular}{|c|c|}
\hline Parameter & Value \\
\hline Detector & n-bulk, p-strip, single-sided, AC-coupled Si $\mu$ strip \\
\hline Detector thickness & $410 \mu \mathrm{m}$ \\
\hline Nominal Energy Range & $15-45 \mathrm{keV}$ \\
\hline Energy Resolution & $\sim 8 \mathrm{keV}$ FWHM \\
\hline Geometric Area & $1344 \mathrm{~cm}^{2}$ \\
\hline Effective Area (max) & $230 \mathrm{~cm}^{2}$ \\
\hline Field of View & $107^{\circ} \times 68^{\circ}$ \\
\hline Mask Transparency & $50 \%$ \\
\hline Angular Resolution & 6 arcmin \\
\hline Mask Element Size & $242 \mu \mathrm{m}$ \\
\hline Detector Strip Size & $121 \mu \mathrm{m} \times 190 \mathrm{~mm}$ \\
\hline Mask-Detector Distance & $142 \mathrm{~mm}$ \\
\hline Timing Resolution & $2 \mu \mathrm{s}$ \\
\hline Timing Accuracy & $5 \mu \mathrm{s}$ \\
\hline Point Source Location Accuracy & $1-2 \operatorname{arcmin}$ \\
\hline Point Source Sensitivity & 15 mCrab (1D) \\
\hline Data Transmission & Event-by-Event, 32-bit/event \\
\hline
\end{tabular}


Table 2

Measured alignment between the outermost individual detector strips and their reference mask strips. The uncertainty in this measurement is only due to the optical targeting of the strip, and was estimated as $\sim 10 \mu$ m linear, corresponding to $\sim 10$ arcsec.

\begin{tabular}{|c|c|c|c|}
\hline Detection Unit & Average & Internal & External \\
(coding direction) & $(\operatorname{arcsec})$ & $(\operatorname{arcsec})$ & $(\operatorname{arcsec})$ \\
\hline D0 (Z) & +18.0 & +14.4 & +21.6 \\
D1 (X) & -18.0 & -18.0 & -18.0 \\
D2 (Z) & -46.8 & -50.4 & -43.2 \\
D3 (X) & +14.4 & +7.2 & +25.2 \\
\hline
\end{tabular}


This figure "fig1.jpg" is available in "jpg" format from: http://arxiv.org/ps/0708.0123v1 
This figure "fig2.jpg" is available in "jpg" format from: http://arxiv.org/ps/0708.0123v1 
This figure "fig3.jpg" is available in "jpg" format from: http://arxiv.org/ps/0708.0123v1 
This figure "fig4.jpg" is available in "jpg" format from: http://arxiv.org/ps/0708.0123v1 
This figure "fig5.jpg" is available in "jpg" format from: http://arxiv.org/ps/0708.0123v1 
This figure "fig6.jpg" is available in "jpg" format from: http://arxiv.org/ps/0708.0123v1 
This figure "fig7.jpg" is available in "jpg" format from: http://arxiv.org/ps/0708.0123v1 
This figure "fig8-9.jpg" is available in "jpg" format from: http://arxiv.org/ps/0708.0123v1 
This figure "fig10.jpg" is available in "jpg" format from: http://arxiv.org/ps/0708.0123v1 
This figure "fig11.jpg" is available in "jpg" format from: http://arxiv.org/ps/0708.0123v1 
This figure "fig12-13.jpg" is available in "jpg" format from: http://arxiv.org/ps/0708.0123v1 
This figure "fig14-15.jpg" is available in "jpg" format from: http://arxiv.org/ps/0708.0123v1 
This figure "fig16.jpg" is available in "jpg" format from: http://arxiv.org/ps/0708.0123v1 
This figure "fig17.jpg" is available in "jpg" format from: http://arxiv.org/ps/0708.0123v1 
This figure "fig18.jpg" is available in "jpg" format from: http://arxiv.org/ps/0708.0123v1 
This figure "fig19.jpg" is available in "jpg" format from: http://arxiv.org/ps/0708.0123v1 
This figure "fig20.jpg" is available in "jpg" format from: http://arxiv.org/ps/0708.0123v1 
This figure "fig21.jpg" is available in "jpg" format from: http://arxiv.org/ps/0708.0123v1 
This figure "fig22.jpg" is available in "jpg" format from: http://arxiv.org/ps/0708.0123v1 
This figure "fig23.jpg" is available in "jpg" format from: http://arxiv.org/ps/0708.0123v1 